\section{SOI: 1.1/TAS DOI: 10.15863/TAS International Scientific Journal Theoretical \& Applied Science}

p-ISSN: 2308-4944 (print) e-ISSN: 2409-0085 (online)

Year: 2018 Issue: $06 \quad$ Volume: 62

Published: $26.06 .2018 \quad$ http://T-Science.org

SECTION 4. Computer science, computer engineering and automation.
Vadim Andreevich Kozhevnikov

Senior Lecturer,

Peter the Great St.Petersburg Polytechnic University

Oleg Yurievich Sabinin

Candidate of Engineering Sciences,

Peter the Great St.Petersburg Polytechnic University

Julia Efimovna Shats

Student,

Peter the Great St.Petersburg Polytechnic University

UDC 004.4'2

\title{
DEVELOPMENT OF CROSS-PLATFORM UTILITY FOR THE DBA USING A LIBRARY FOR WORKING WITH BOTS
}

Abstract: This article considers the basic principles of building cross-platform utility for monitoring of the database state. The information about collection of database statistics and development of the library for implementing of bots on platforms of various messengers is analyzed.

Key words: Databases, Data systems.

Language: English

Citation: Kozhevnikov VA, Sabinin OY, Shats JE (2018) DEVELOPMENT OF CROSS-PLATFORM UTILITY FOR THE DBA USING A LIBRARY FOR WORKING WITH BOTS. ISJ Theoretical \& Applied Science, 06 (62): 127-131.

Soi: http://s-o-i.org/1.1/TAS-06-62-23 Doi: crossef https://dx.doi.org/10.15863/TAS.2018.06.62.23

\section{Introduction}

At the present time there are many problems faced by administrators of databases (DBs) and database management systems (DBMS). One of the main problems is the problem of data security. For DBMS, which store and manage the data of enterprises, the task of ensuring safety is paramount.

By conducting a set of measures aimed at data protection, database administrators (DBAs) try to protect them as much as possible and to limit the scope of ordinary users, but for various reasons they can still create a wide variety of issues.

The article discusses the creation of a database administrator utility, based on a messaging bot, the main task of which is to notify the administrator about attempts to connect to the database and change it.

\section{Motivation}

Any productional database is constantly changing, both in terms of internal state (addition and modification of data), and in terms of external conditions associated, for example, with the development of software to meet new requirements. The qualitative work of the database is critically dependent on correct and timely diagnosis. It is important not only to know all the information about the system, but also to learn about the changes as quickly as possible. You must constantly monitor the operation of the database to ensure that it is working correctly and it is not suspended by running a complex task that reduces performance. The database administrator should receive notifications about:

- How the database currently works - in general and in comparison, with other periods;

- Which requests are most heavily burdened by the server (CPU, memory, storage systems);

- How many requests and which ones have been finished, at what time and by which users

- etc.

\section{Implementation}

It is proposed to create a bot based on instant messenger bot library, which will allow the DBA to be constantly able to check the status of the system and be aware of its changes due to a set of commands and push notifications. This solution seems to be the most correct, since manual testing takes a lot of time, while notification of emergency situations (using push technology [1]) and the ability to quickly learn about operations can significantly reduce time costs. It should also be noted that a lot of organizations use bots, and today they are available on any platform, whether it's a desktop computer, a mobile phone or a web browser. 
As messengers for the implementation of the utility, were selected:

- Slack, the most popular corporate messenger; [2]

- Facebook Messenger, the most popular messenger around the world; [3] [4].

- Telegram, the best platform for creating bots

Since the implementation of such functionality for different DBMS is a laborious process, within this article it was decided to implement the support of only one of them - PostgreSQL, as this DBMS is free, advanced, open and rapidly gaining popularity, replacing competitors (including powerful commercial DBMSs such as Oracle, MS SQL Server, DB2, etc.). [5; 6]

The Bot API consists of several modules:
- Bot Library - a library for interacting with various messengers (Facebook Messanger, Telegram, Slack);

- Pg Log Module - module for working with PostgreSQL log-files. It takes information about the actions that occur with the database from the file, analyzes it for error messages to issue a notification to the administrator as soon as possible about what is happening;

- Pg Stats Module - a module for working with statistics, which provides PostgreSQL using the pg_stat views. This module makes queries to the extensions pg_stat_activity and pg_stat_statements and sends data to end users using the Bot Library.

The component scheme of the program is shown in Fig.1.

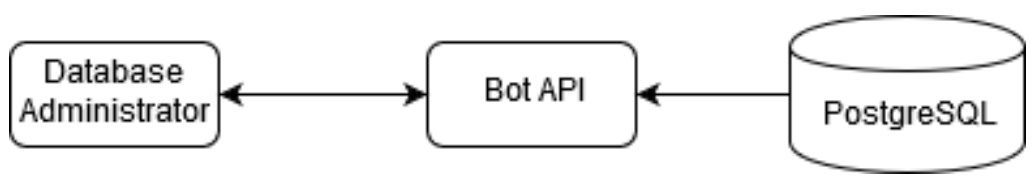

Fig. 1. Component scheme

\subsection{Collecting database statistics}

In PostgreSQL DBMS, there are several ways to collect information about the database [7], which we will use to send to the administrator through the bot. The main source of this information is the pg_stat_statements extension. It is a set of procedures for obtaining various statistics about the queries that the server processes. In order to obtain and process statistics, this module uses a pg_stat_statements view and auxiliary functions Pg_stat_statements_reset and pg_stat_statements. Based on the obtained statistics the administrator can perform request monitoring — look at the statistics on time. This is extremely useful for finding the causes of various problems and for understanding what is happening on the database server in general.

While using pg_stat_statements extension the administrator receives the grouped statistics not for each query but for a group of queries identical in terms of PostgreSQL. Besides query information with help of this set of views you can learn about the number and time of reading and writing blocks on the disk (Table 1 [8]). All counters keep statistics from the start or from the moment they are reset by the database administrator.

\section{Variables that contain information about the database}

Table 1.

\begin{tabular}{|l|l|}
\hline \multicolumn{1}{|c|}{ Name } & \multicolumn{1}{|c|}{ Description } \\
\hline userid and dbid & OID of user and database who executed the statement \\
\hline queryid & Internal hash code, computed from the statement's parse tree \\
\hline query & Text of a representative statement \\
\hline calls & Number of times executed \\
\hline total_time & Total time spent in the statement, in milliseconds \\
\hline min_time, max_time and mean_time & $\begin{array}{l}\text { Minimum, maximum and mean time spent in the statement, in } \\
\text { milliseconds }\end{array}$ \\
\hline stddev_time & $\begin{array}{l}\text { Population standard deviation of time spent in the statement, in } \\
\text { milliseconds }\end{array}$ \\
\hline rows & Total number of rows retrieved or affected by the statement \\
\hline $\begin{array}{l}\text { shared_blks_hit, } \\
\text { shared_blks_read, } \\
\text { shared_blks_dirtied and }\end{array}$ & $\begin{array}{l}\text { Total number of shared block cache, read, dirtied and written } \\
\text { hits by the statement }\end{array}$ \\
\hline
\end{tabular}




\begin{tabular}{|l|l|}
\hline shared_blks_written & \\
\hline $\begin{array}{l}\text { local_blks_hit, local_blks_read, } \\
\text { local_blks_dirtied and } \\
\text { local_blks_written }\end{array}$ & $\begin{array}{l}\text { Total number of local block cache, read, dirtied and written } \\
\text { hits by the statement }\end{array}$ \\
\hline $\begin{array}{l}\text { temp_blks_read and } \\
\text { temp_blks_written }\end{array}$ & Total number of temp blocks read and written by the statement \\
\hline \begin{tabular}{l} 
blk_read_time and blk_write_time \\
\hline
\end{tabular} & $\begin{array}{l}\text { Total time the statement spent reading and writing blocks, in } \\
\text { milliseconds }\end{array}$ \\
\hline
\end{tabular}

It should be mentioned that only completed queries fall in pg_stat_statements. That is, if the query runs for a long time and is still not finished, it will be visible only in the pg_stat_activity view.

Also, information about database users and their transactions can be obtained from PostgreSQL log-files (Table 2 [9]). In order to convert information and output it to user, log-files should be searched to find queries that are of interest to the administrator (user of the utility).

Log-messages PostgreSQL

Table 2.

\begin{tabular}{|l|l|}
\hline \multicolumn{1}{|c|}{ Severity } & \multicolumn{1}{c|}{ Usage } \\
\hline DEBUG1..DEBUG5 & Provides successively-more-detailed information for use by developers. \\
\hline INEO & $\begin{array}{l}\text { Provides information implicitly requested by the user, e.g., output from VACUUM } \\
\text { VERBOSE. }\end{array}$ \\
\hline NOTICE & $\begin{array}{l}\text { Provides information that might be helpful to users, e.g., notice of truncation of long } \\
\text { identifiers. }\end{array}$ \\
\hline WARNING & Provides warnings of likely problems, e.g., COMMIT outside a transaction block. \\
\hline ERROR & Reports an error that caused the current command to abort. \\
\hline LOG & Reports information of interest to administrators, e.g., checkpoint activity. \\
\hline FATAL & Reports an error that caused the current session to abort. \\
\hline PANIC & Reports an error that caused all database sessions to abort. \\
\hline
\end{tabular}

\subsection{Development of the Bot Library module}

At the core of the library programming for working with bots is the understanding that the web server installs the so-called webhooks - the addresses to which the messenger server will send information about events in the chat with the users. In fact, the server receives a message and returns the response that the developer has written to user. Therefore, it is possible to consider that the most part of the bot's software interface is about to receive and send information in JSON format to special addresses on the server of the messenger.

To develop the module, it was decided to use the Java language because it is one of the most popular programming languages [10] and its library standard has everything necessary to implement the interaction with messengers [11]. Using only standard classes and methods involves the need to write a lot of repetitive low-level code, so it is more convenient to use libraries that simplify interaction with messengers. In order to do this a set of libraries which is the part of the Bot library was implemented.

To ensure that the libraries that were written can be used in other projects you should use the framework to automate the assembly (such as Maven or Gradle) to connect external dependencies from repositories such as Maven Repository [12]. That is why this approach was used to conveniently connect the implemented library.

Once the libraries were created and built, the main task was to merge them into the Bot Library in order to developer could immediately transmit his bot to multiple platforms [11].

\subsection{Analysis and testing}

On average, to get information about the status of the database, the administrator is forced to: resort to either using a paid software package, or manually to view log-files and make requests for views and packages.

Due to the implemented solution, many administrators can have a significant increase in productivity.

To check how utility is running, there was created a database that stores data to execute test queries for receiving notifications. It was decided to use Open Server as a server and the software supplied with it, which provides a graphical interface 
to operate with database - PgAdmin that improves the process of setting up the environment for development. The number of transactions were executed by the premade users, also some errors were emulated for checking push notifications. As an illustration, in Fig. 2 you can see the administrator's query to search executed "select" statements and the result of this search on different platforms.

Therefore, due to receiving notifications or requests to the bot the administrator can have up-todate information about the status of the database, users, executed scripts, errors, etc.

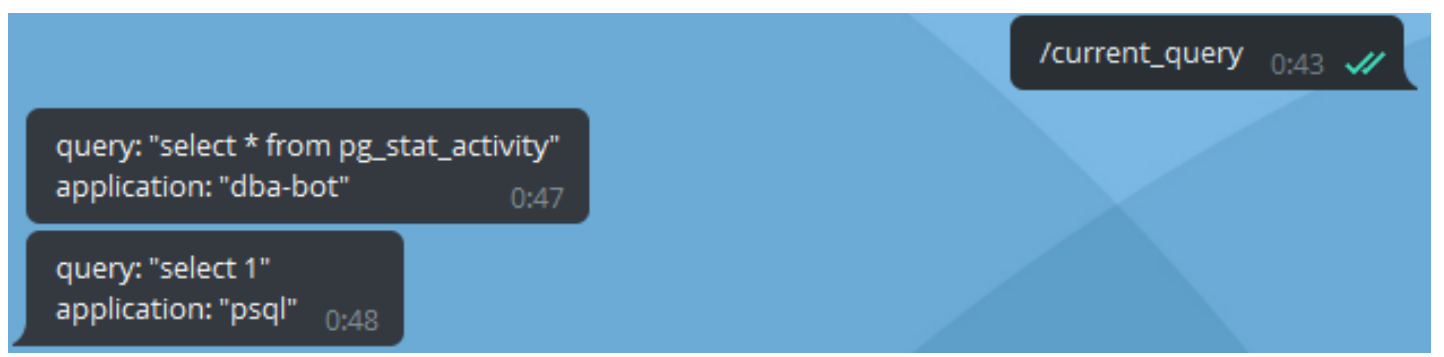

Fig. 2. Checking completed database requests on Slack platforms.

\section{Conclusion}

Based on methods of construction that were offered in article the utility allows DBA to quickly, easily search information about the status of the database, usage statistics, and via push notifications instantly receive information about critical situations. The architecture offered above has an important advantage - its cross-platform that gives you a possibility to use the implemented system on a wide range of devices (clients on the desktop computer, the web browser, the mobile phone).

The implemented library allows users to create basis bots of any content on several platforms at once that increases development speed.

In the future, the authors plan is to improve the created products by adding new messengers, graphs of impact on server load and other features.

\section{References:}

1. (2018) Introduction to Push Notifications. [online] Google Developers. Available at: https://developers.google.com/web/ilt/pwa/intro duction-to-push-notifications [Accessed 9 April 2018]

2. J. Duffy (2018) The Best Business Messaging Apps of 2018. [online] PCMAG. Available at: https://www.pcmag.com/roundup/355674/thebest-team-messaging-apps [Accessed 20 Jan. 2018].

3. (2018) Statista, Most popular messaging apps 2018. [online] Statista. Available at: https://www.statista.com/statistics/258749/most -popular-global-mobile-messenger-apps/ [Accessed 28 Jan. 2018].

4. (2018) Telegram, Telegram Bot API. [online] Telegram. Available at: https://core.telegram.org/bots/api [Accessed 16 Jan. 2018].
5. L. Smith (2017) What PostgreSQL has over other open source SQL databases: Part I. [online] Compose Articles. Available at: https://www.compose.com/articles/whatpostgresql-has-over-other-open-source-sqldatabases/ [Accessed 15 Dec. 2017].

6. L. Smith (2017) What PostgreSQL has over other open source SQL databases: Part II. [online] Compose Articles. Available at: https://www.compose.com/articles/whatpostgresql-has-over-other-open-source-sqldatabases-part-ii/ [Accessed 15 Dec. 2017].

7. S. Riggs, G. Ciolli, G. Bartolini G., H. Krosing (2015) PostgreSQL 9 Administration Cookbook - Second Edition. Packt Publishing, 2015.

8. (2017) PostgreSQL Documentation. [online] PostgreSQL. Available at: https://www.postgresql.org/docs/9.1/static/runti me-config-logging.html [Accessed 30 Dec. 2017]. 
\begin{tabular}{l|lr|ll|ll} 
& ISRA (India) & $=\mathbf{1 . 3 4 4}$ & SIS (USA) & $=\mathbf{0 . 9 1 2}$ & ICV (Poland) & $=\mathbf{6 . 6 3 0}$ \\
Impact Factor: & ISI (Dubai, UAE) $=\mathbf{0 . 8 2 9}$ & PUH (Russia) $=\mathbf{0 . 2 0 7}$ & PIF (India) & $=\mathbf{1 . 9 4 0}$ \\
& GIF (Australia) & $=\mathbf{0 . 5 6 4}$ & ESJI (KZ) & $=4.102$ & IBI (India) & $=\mathbf{4 . 2 6 0}$ \\
& JIF & $=\mathbf{1 . 5 0 0}$ & SJIF (Morocco) & $=\mathbf{2 . 0 3 1}$ & & \\
\hline
\end{tabular}

9. Takahiro, P. (2017) Geoghegan. PostgreSQL Documentation. [online] PostgreSQL.

Available at:
https://postgrespro.ru/docs/postgrespro/9.5/pgst atstatements\#/ [Accessed 28 Dec. 2017].

10. B. Putano (2018) Most Popular and Influential Programming Languages of 2018. [online] Stackify. Available at: https://stackify.com/popular-programminglanguages-2018/ [Accessed 28 Dec. 2017].
11. V. A. Kozhevnikov, O. Y. Sabinin, J. E. Shats, (2017) Library development for creating bots on Slack, Telegram and Facebook messengers, ISJ Theoretical \& Applied Science, 06 (50), p. 59-62, 2017.

12. T. O'Brien, Van Zyl, J..Maven (2009) The Definitive Guide, O'Reilly Media Inc., Sebastopol, 2009. 